\title{
Échec et mat! Constat d'échec et imprécation contre le triomphalisme du storytelling chez Fernando Vallejo
}

Lionel Souquet

\section{OpenEdition}

Journals

Édition électronique

URL : https://journals.openedition.org/cher/11961

DOI : 10.4000/cher.11961

ISSN : 2803-5992

Éditeur

Presses universitaires de Strasbourg

Édition imprimée

Date de publication : 30 juin 2012

Pagination : 13-25

ISBN : 978-2-35410-046-9

ISSN : 1968-035X

Référence électronique

Lionel Souquet, « Échec et mat ! Constat d'échec et imprécation contre le triomphalisme du storytelling chez Fernando Vallejo », reCHERches [En ligne], 8 | 2012, mis en ligne le 21 février 2022, consulté le 23 février 2022. URL : http://journals.openedition.org/cher/11961; DOI : https://doi.org/10.4000/cher. 11961

\section{(ब) $(0 \odot$}

Ce(tte) œuvre est mise à disposition selon les termes de la Licence Creative Commons Attribution -

Pas d'Utilisation Commerciale - Partage dans les Mêmes Conditions 4.0 International. 


\title{
Échec et mat ! \\ Constat d'échec et imprécation contre le triomphalisme du storytelling chez Fernando Vallejo
}

\author{
LIONEL SOUQUET \\ Université de Bretagne Occidentale (Brest)
}

Lo más rescatable de Colombia eran sus vicios, pues por lo demás era un país vencido, fracasado, podrido.

Podrido de envidia y odio y con el alma rota. Fernando Vallejo

« $\mathbf{E}_{\text {paradoxe - être l'axiome valléjien, comme semble le confirmer }}^{\text {chec et création" pourrait - par excellence, c'est un premier }}$ la présentation de deux études consacrées à ce talentueux auteur, dans un ouvrage sur l'échec dans la littérature hispano-américaine! La genèse artistique de Fernando Vallejo Rendón, né à Medellín en 1942, semble marquée du sceau de l'échec puisque, à l'instar de l'Argentin Manuel Puig, l'auteur colombien n'est venu à l'écriture qu'en 1985, après avoir constaté le fiasco de sa carrière de cinéaste, amorcée dix-sept ans plus tôt, dès 1968. L'ironie du sort voudra, d'ailleurs, qu'il accède à la notoriété en 2000, grâce à l'adaptation cinématographique de son roman La virgen de los sicarios (1994), par un autre réalisateur, Barbet Schroeder!

Nous verrons donc comment est né et s'est développé, chez Vallejo, un processus de création littéraire intrinsèquement lié à l'avortement de sa vocation cinématographique et comment le récit souvent autofictionnel et faussement narcissique de cet échec est devenu la matière même de son écriture, prenant la forme d'un leitmotiv thématique où se déclinent plusieurs méditations sur l'échec personnel et collectif. Départicularisant 
son expérience intime de l'échec, Vallejo lui donne une valeur parabolique, soutenue par la puissance subversive d'une ligne néo-picaresque qui traverse toute son œuvre. Le thème de l'échec interroge la société et ses mythes triomphalistes.

On ne peut qu'être frappé par la fascination de Vallejo - personnage polémique s'il en est - pour les grandes figures de poètes et d'intellectuels «maudits» tels que le Colombien Porfirio Barba Jacob ou Martin Heidegger, ostracisés après avoir été portés aux nues. Nous verrons enfin que l'œuvre du philosophe allemand, et tout particulièrement sa Lettre sur l'humanisme, publiée après le cataclysme de la Seconde Guerre mondiale, éclaire indirectement mais puissamment le sens philosophique de l'échec dans l'œuvre de Fernando Vallejo.

\section{De l'échec du cinéaste à l'écriture de l'échec}

Vallejo se lance dans la réalisation, dès 1968, avec un court-métrage intitulé Un hombre y un pueblo. Suivront un autre court et surtout trois longmétrages: Crónica roja (1977), En la tormenta (1980) et Barrio de campeones (1981). Crónica roja raconte l'histoire des frères Barragán, deux jeunes gens massacrés par la police dans un quartier du sud de Bogotá. Vallejo obtient un financement mexicain pour Crónica roja, qu'il veut filmer en Colombie, mais doit finalement le réaliser au Mexique (malgré les difficultés pour les décors) à cause des nombreux obstacles et des problèmes de censure plus ou moins explicite qu'un projet aussi dérangeant rencontre dans son pays:

Entonces regresé a Bogotá a tratar de filmarla con el dinero mexicano. ¡Imposible! Ahí estaba el Incomex ${ }^{1}$ para impedirme importar el negativo y los equipos; la Dirección de Tránsito para no darme los permisos que necesitaba para filmar en las calles; el Ministerio de Relaciones Exteriores para no darme las visas de los técnicos que tenía que traer de México; la policía para no darme su protección durante el rodaje y el permiso de que mis actores usaran uniformes como los suyos y pistolas de utilería pues había policías en mi historia... ${ }^{2}$

1 Incomex: Institut Colombien du Commerce Extérieur.

2 «Fernando Vallejo renuncia a la nacionalidad colombiana», texte par lequel Vallejo explique sa décision de renoncer à la nationalité colombienne, diffusé sur Caracol Radio (Cadena Radial Colombiana S.A.), le 8 mai 2007 et disponible sur différents sites et blogs dont Revista Semana.com, Fecha: 05/07/2007: http://www.semana.com/wf_InfoArticulo. aspx?idArt $=103098$ 
Le film suivant, En la tormenta, développe le thème obsessionnel de Vallejo: la "période de la violence» en Colombie (1948-1960). Même enchaînement de déconvenues: il doit faire le film au Mexique et, une fois terminée, l'œuvre est interdite en Colombie. Avec Barrio de campeones, Vallejo change de cadre: le film raconte l'histoire d'un boxeur, issu des quartiers miséreux de Mexico, qui tente de devenir champion pour résoudre les problèmes économiques de sa famille. Mais Vallejo a compris que cette voie était sans issue pour lui et il abandonne le cinéma, tirant plus tard cette amère conclusion: «[...] mis películas, ¿que en qué cinemateca están? En la más completa, la del olvido» (Vallejo 1993: 75-76). Nous disposons de très peu d'éléments objectifs - non fictionnalisés - pour reconstituer les étapes de sa carrière de cinéaste: comme toujours, les faits biographiques restent sous le contrôle quasi exclusif du narrateur autofictionnel. Dans Entre fantasmas, Fernando présente son désir de cinéma comme une sorte de mission messianique: «[...] no me pienso morir sin hacer una película para denigrar de Colombia, una al menos. Para eso nací» (24). Mais, comme nous venons de le voir, la réalisation de ses films sera, à chaque fois, un véritable parcours du combattant. Selon ses dires, plus de vingt ans s'écoulent entre la conception mentale de En la tormenta et sa réalisation:

Saliendo del consulado colombiano en Roma, por el piazzale Flaminio, se me ocurrió la película. La vi completa, con sus decapitados, con sus incendios, con su rencor y su furia, con todo su horror, en un instante de iluminación o alucinación que abarcaba a Colombia. Veintinosecuantos años la llevé en el corazón hasta que pude filmar, por fin, en México, reconstruyendo a Colombia sobre el imposible, y nació como tenía que nacer: a medias, obsoleta y vieja (42).

Comme toujours, Vallejo nous mène en barque. Après avoir feint l'autocritique il retourne ironiquement son constat d'échec en symptôme du dysfonctionnement de la société colombienne: si ses films sur la Colombie sont dépassés, ce n'est pas son talent de cinéaste qui est en cause, c'est la réalité: "¿Qué era eso de esos pelagatos descalzos matando con machete! Colombia hoy mata con ametralladoras, bobito, tontito, ponte al día y botas. Taratatatatá» (42).

Alors qu'il semble vouloir s'en tirer par une pirouette, Vallejo «recycle» des éléments autobiographiques devenus presque anecdotiques (ses échecs en tant que cinéaste) en leur donnant à la fois une portée plus générale et allégorique mais aussi un sens plus profond qui rappelle certains aspects de la parabole. Peu importe, finalement, son échec puisqu'il est devenu un grand écrivain! Ce que le lecteur retient, ce sont les raisons (directes ou indirectes, 
réelles ou fictives) de cet échec: une violence apparemment sans fin, qui perdure depuis plus de soixante ans, qui paralyse toute initiative (l'échec de Vallejo reflète l'absence de tout cinéma colombien) et qui est aussi alimentée par un négationnisme absurde qui apparaît clairement, toujours selon le narrateur autofictionnel, dans les arguments de la commission de censure interdisant la diffusion de son film: «[...] me la prohibió la censura: que era una apología al delito, una incitación a la violencia, una mentira, que Colombia no era así. Allá todos morían a los ciento veinte años de viejos en su cama, tristes de irse pero felices por haber vivido» (Vallejo, Años de indulgencia, 1993: 82).

Reconstituant le parcours de cinéaste de Vallejo, notamment à partir de ce que l'auteur en dit dans Entre fantasmas et dans Años de indulgencia, Javier Murillo arrive à la conclusion suivante:

Vallejo y su frustración forman también parte del fracaso cinematográfico colombiano. Y él, que se había ido para contar, no tiene ya ninguna duda de que pertenece a ese país que quería mostrar [...] Buscó el cine y fracasó, pero mientras buscaba vivió y mientras vivió escribió. [...] La evidencia del lenguaje verbal en vez del cinematográfico transforma la mirada; si al principio de su viaje escribía mientras buscaba la imagen visual, al final la escritura en sí misma es el centro de la búsqueda.

Vallejo explique lui-même comment ses piétinements en tant que cinéaste l'ont amené à chercher, pendant dix ans, un dérivatif dans l'écriture de $E l$ Mensajero (1991), la biographie de l'écrivain colombien Porfirio Barba Jacob dont les succès littéraires parviennent à peine - selon Vallejo - à illuminer une vie rythmée d'échecs et de disgrâces. L'effet de miroir entre la biographie de Barba Jacob et ce que Vallejo nous dit de lui-même est si saisissant que $E l$ Mensajero prend souvent des accents autobiographiques. Rien d'étonnant à ce que le premier roman de Vallejo, Los días azules, paraisse en 1985, quatre ans après son dernier film. Le récit de ses déboires cinématographiques vaut donc autant - voire plus - comme matière littéraire que comme témoignage autobiographique et c'est là une des manières les plus géniales de transformer une frustration en création.

\section{Échec de l'action}

Toute tentative de transposition du cinéma dans le domaine de l'écriture est finalement vouée à l'échec quand on a compris que le $7^{\text {e }}$ art était un système prélinguistique. Vallejo sait qu'à part quelques artifices (illusion de panoramique, de zoom, de montage, etc.), le meilleur moyen de mettre du 
cinéma dans la littérature, de nourrir l'écriture de cinéma, c'est de s'en tenir principalement aux concepts: réinjecter et recycler les concepts développés par le cinéma et s'interroger sur le rôle du cinéma comme simulacre générateur de vérité, c'est-à-dire sur l'essence même de l'autofiction. C'est le sens de cette affirmation paradoxale de Vallejo: «He llegado a la conclusión de que el cine es como la novela, un género artificioso, mentiroso [...]» (Vallejo, 1993: 234). Faute de faire du cinéma, Vallejo va tenter de «resémantiser» le cinéma dans la littérature.

Le système de Gilles Deleuze, philosophe de la littérature et du cinéma et penseur de la postmodernité, est donc particulièrement adapté à l'étude d'une telle transposition. Il faut bien comprendre que le travail de Deleuze ne porte pas sur le cinéma mais sur les concepts du cinéma qu'il articule, notamment, avec la philosophie de Bergson et le concept d'image-mouvement développé dans Matière et Mémoire (1896). Deleuze élabore une grande théorie qui, comme le souligne Philippe Mengue (241-272), ne constitue pas une histoire du cinéma mais plutôt une taxinomie, une sorte d' "histoire naturelle» qui classe les signes cinématographiques en deux natures hétérogènes d'images, en deux combinatoires différentes des mêmes éléments: l'image classique (image-mouvement) et l'image moderne (image-temps), le néo-réalisme italien se trouvant à la charnière de ces deux types d'images.

Après le traumatisme de la Deuxième Guerre mondiale et la découverte des camps de concentration, l'action devient impossible face à l'intolérable. Cette rupture provoque alors la disparition d'un cinéma classique basé sur l'action et la narration. Loin d'être devenu "passif», le cinéma révèle et dénonce ce bouleversement en nous montrant des personnages incapables d'agir, des personnages qui regardent l'horreur ou même la splendeur du monde, souvent du « $[\ldots]$ regard perdu de l'être absent au monde autant qu'à soi [...]» (Deleuze 1985: 17) comme le "héros» autofictionnel de Vallejo dans El fuego secreto: "[...] así esté borracha y bajo una vela y vea a tientas como veo yo desde que me robaron las gafas [...]» (136) ou dans la Rambla paralela: «[...] el viejo se puso a recordar con los ojos abiertos, viendo sin ver, en el aire [...]» (41). Un peu plus loin, le narrateur explique le «point de vue» de son double - son "point de vue» donc - : «Contuvo la respiración, cerró los ojos y por entre la barbaridad de carros se cruzó a ciegas el Paralelo, la calle de su recuerdo. Incólume llegó a la otra orilla, a la acera opuesta diciéndose que él para ver ya no necesitaba ver, que con lo visto en su larga vida había visto bastante» (46). La faiblesse de la vue est la métaphore d'une lucidité aveuglante. C'est alors que l'identification se renverse, le personnage 
est devenu une sorte de spectateur, un «voyant»: «Quand l'action est devenue impossible ou barrée, suspendue, on devient un voyant, "c'est un cinéma de voyant", dit Deleuze, qui supprime toute narration» (Mengue 1994: 246).

Il y a, en effet, dans les romans de Vallejo, une complète remise en question de la fonction narrative et de l'action ainsi qu'un double renoncement à l'image-mouvement (non seulement par le passage de l'image au mot mais aussi par le choix d'exprimer en mots l'immobilité et l'inaction). Alors que toute action semble vouée à l'échec, le discours s'impose comme seul élément constructif et comme fin en soi. La logorrhée du personnage autofictionnel prend le pas sur le geste et le remplace presque complètement: «[...] era un disco rayado, tocando siempre la misma, la misma, la misma canción» (2002: 91). On constate le même immobilisme - compensé par de puissantes lignes de nomadisme - dans La virgen de los sicarios où Fernando - littéralement médusé par l'horreur de la réalité - regarde les sicaires tuer leurs victimes. Le narrateur autofictionnel, notamment, passe plus de temps à parler qu'à agir, frappé d'une sorte d' «impuissance» liée à sa soi-disant vieillesse cacochyme: en 1985, dans Los caminos a Roma, Vallejo qui n'a alors que quarante-trois ans se décrit déjà comme «Un pobre viejo con reuma que ni se puede mover» (114). Huit ans plus tard, à cinquante-etun ans, en 1993, dans Años de indulgencia, le narrateur autodiégétique se présente comme «un viejo anecdotero [...] vejete maldiciente, quebradizo, saco de huesos [...]» (61). Le thème du Carpe diem est d'ailleurs rapidement explicité par une évocation de Ronsard (62). Bien qu'il soit relativisé par l'ironie, l' «autoportrait en vieillard» finit par s'imposer par sa récurrence. Le leitmotiv du barbon valétudinaire ne manque pas de frapper le lecteur habitué au "jeunisme» de la culture médiatique postmoderne et à son mythe de la jeunesse éternelle. Dans une société où le cinéma et la publicité nous abreuvent de héros toujours jeunes et beaux, emblèmes de l'idéologie triomphaliste du winner, ces narrateurs et personnages vieillis avant l'heure offrent une figure presque insolite de loser ou d'anti-héros en rupture avec l'agitation du monde. Beauté et jeunesse, dont l'éloge est permanent, appartiennent désormais au passé ou à l'altérité.

\section{Auto-dégradation néo-picaresque et mise en échec de l'honorabilité familiale}

Fils de grands bourgeois (son père est un célèbre politicien conservateur), «héritier» notoire, Vallejo se fabrique ironiquement une hérédité «négative» et renverse la notion même de déterminisme en inscrivant - par le biais du 
récit autofictionnel - la généalogie et l'histoire familiale dans une perspective néo-picaresque particulièrement originale. Alors que l'autobiographe classique se présente souvent en majesté et semble toiser le monde, le point de vue néo-picaresque est celui d'une "vision d'en bas " puisque le pícaro - le gueux - est (presque) toujours de basse extraction: ses parents, ouvertement marginaux ou délinquants, représentent la lie de la société. Tandis que le «vrai» pícaro croit s'élever en visant la respectabilité due à l'argent et aux apparences, le narrateur autofictionnel de Vallejo suit une puissante ligne de fuite créatrice qui l'arrache à la molarité/moralité œedipienne du Bildungsroman familial. Se livrant à une entreprise de destruction de la respectabilité bourgeoise de sa famille, Vallejo brosse, avec son humour au vitriol, des portraits parodiques et dégradés de ses proches. Dès les premières pages de Los días azules, il représente son grand-père comme un hurluberlu écraseur de piétons (un déjanté au sens propre) et, dans El desbarrancadero, sa mère - qu'il surnomme "la Folle» - apparaît comme une castratrice et dangereuse hystérique. D’une implacable intransigeance, ses autofictions lui permettent d'explorer la - sa - famille comme noyau névrotique et explosif, au cœur d'une société chaotique. L'échec de la famille devient aussi la métaphore de l'échec de la société.

Les caractéristiques topo- (et hydro-)graphiques de la propriété familiale rapprochent étonnamment l'autofictionneur valléjien de la figure picaresque de Lazarillo, né - selon ses dires - dans le fleuve Tormes. Chez l'auteur colombien, pour qui les maisons d'enfance sont un thème récurrent, obsessionnel, la demeure - pourtant bourgeoise, voire aristocratique - se conjugue curieusement au schème du cloaque. Inéluctablement, la famille de Fernando s'enlise dans une propriété qui se transforme en marécage: «[...] el agua se empezó a filtrar también a las huertas, a las pesebras, a la casa, a los potreros, a toda la propiedad. Y Santa Anita [...] quedó sirviendo para un carajo, se volvió un pantano. Clavábamos un palo de escoba en el pasto y brotaba un surtidor» (1985a: 53). Cette image régressive est prolongée par l'évocation, dans plusieurs de ses romans, du Cauca, l'un des fleuves les plus importants de Colombie, qui baigne la ville de Medellín, invariablement décrit comme un égoût (ce qu'il est, d'ailleurs, réellement), rejoignant les délires scatologiques de la picaresque quévédienne: «El río, turbulento, se llama el Cauca, y entre tantos secretos de sus honduras tiene una « $\mathrm{u}$ » en el medio» (1986: 224).

Mais c'est surtout en exhibant son homosexualité décomplexée et celle de ses frères fumeurs de marijuana que Vallejo parvient à saper la notabilité 
et la respectabilité sociale de sa famille, décentrant la norme hétérosexuelle vers une homosexualité activement "perverse», "déviante», irrécupérable et subversive (rétive à toute normativité puisque l'autofictionneur évoque aussi ses penchants pédophiles). Le «nomadisme» sexuel (et générique) des personnages de Vallejo - et notamment des doubles autofictionnels prend donc une connotation hautement philosophique et politique. Dans cette famille de notables conservateurs, l'homosexualité apparaît comme un échec de la tradition, de la filiation et de la transmission des valeurs bourgeoises. Cette idée de rupture et d'échec est encore accentuée, dans $E l$ desbarrancadero, par le récit de l'agonie du frère de l'autofictionneur, Darío, malade du sida, et l'évocation du suicide d'un autre frère, Silvio. Cet échec de la transmission généalogique est donc revendiqué comme une rupture nécessaire.

Au lieu de clamer l'innocence des dominés sociaux, dont il considère faire partie en tant qu'homosexuel, l'autofictionneur valléjien endosse et devance l'opprobre, s'accusant des pires crimes et dressant, avec une ambiguité jouissive, un étonnant (auto-)portrait de l'artiste en assassin. Embarqué dans un enchaînement d'actes criminels dont la logique est hallucinatoire, l'autofictionneur de Los caminos a Roma déclare avoir commis deux crimes gratuits. Vallejo cultive un paradoxe: rejetant l'image finalement trop normative de l'artiste engagé - dont Sartre serait le parangon: "Ya iba a decirle a Sartre que no compartía su tesis del compromiso, que el único compromiso que yo aceptaba era el del hombre consigo mismo [...]» (1985b : 23) -, c'est sa propre vie qu'il prétend engager dans son œuvre.

\section{L'échec de l'humanisme}

La figure de l'artiste-criminel soulève aussi la question de la culpabilité commune de l'humanité: «[...] todos somos culpables» (2001: 34). Imprécateur et provocateur mais refusant la tautologie moralisante de ceux que Sloterdijk appelle les «humanitaristes», Vallejo agace les complices d'une société hypocrite et cynique qui, s'accommodant d'un discours "politiquement correct» et d'un "vernis égalitaire-égalisateur», ignore les différences, oublie les laissés pour compte et exploite les plus faibles. Le monde, derrière ses grandes déclarations de principes non tenues - la Démocratie, les Droits de l'Homme et «l'Humanitaire» - ne connaît, en fait, que «la loi du plus fort» (pour paraphraser Fassbinder). Dans Mi hermano el alcalde, roman qu'il qualifie de "chronique», Vallejo présente la ville de Támesis, dont son frère fut réellement maire de 1998 à 2000, comme 
un univers néo-picaresque, un microcosme de la société colombienne où la démocratie est gangrénée et mise en échec par la corruption et dont les politiciens et les "honnêtes" citoyens sont finalement plus cyniques encore que les tueurs à gages de La virgen de los sicarios:

Candidato que no promete no llega. Y alcalde que cumple como Carlos es, cual se dice en Colombia la sabia, un güevón. Créanmelo. Cuando lleguen al poder embólsense lo que puedan y gástenselo en lo que sea: en putas, en yates, en compact discs.

$[\ldots]$

Y me dirá que la política ensucia y corrompe más que la cocaína o coca, y no le falta razón. [...]

Cuarto Mandamiento: no te hagas elegir si no vas a robar, pendejo. Y que el pueblo trague polvo y coma mierda $(2004: 31-32,85,105)$.

Le culte de la figure de l'artiste criminel explique partiellement la fascination de Vallejo pour Heidegger puisqu'après avoir été considéré comme l'un des plus grands penseurs du $\mathrm{XX}^{\mathrm{e}}$ siècle, le philosophe allemand sera mis au ban de la société intellectuelle pour s'être compromis avec le régime nazi, ce qui le rendait implicitement complice de l'un des systèmes les plus criminels de l'histoire de l'humanité. La culpabilité, complicité ou complaisance de Heidegger reste, jusqu'à nos jours, un sujet fort polémique, comme le prouvent les prises de position de nombreux intellectuels. Après Hannah Arendt, René Char, George Steiner ou Elisabeth Roudinesco, c'est le philosophe allemand Peter Sloterdijk qui sera à l'origine de l'un des plaidoyers les plus intéressants en faveur d'une réhabilitation intellectuelle de Heidegger.

C'est à l'automne 1946 que Heidegger a écrit sa célèbre Lettre sur l'humanisme, d'abord adressée à Jean Beaufret à Paris, et par la suite publiée et traduite. Dans ce texte essentiel, Heidegger constate l'échec de l'humanisme et de ses variantes. La philosophie antique, le christianisme, les Lumières, le marxisme et l'existentialisme ne seraient que des «agents de la non-pensée» servant à éluder la véritable question de la nature humaine et l'humanisme serait finalement le complice de toutes les horreurs commises au nom du bien-être de l'humanité. Heidegger voit l'homme comme un animal sauvage domestiqué par l'éducation. Le philosophe se demande donc ce qui pourrait encore domestiquer l'homme si toutes les tentatives d'éducation ont échoué, comme le prouve l'impuissance de l'humanisme face à la barbarie nazie.

En 1999, dans Règles pour le parc humain, une conférence qui fera date et provoquera une importante controverse philosophique, Sloterdijk revient sur le texte de Heidegger en le mettant notamment en perspective avec la pensée 
de Nietzsche. Sloterdijk montre que, dans Ainsi parlait Zarathoustra (18831885), se dissimule déjà un discours théorique sur l'homme comme puissance capable d'apprivoiser et de faire de l'élevage. En outre, Zarathoustra révèle que les prêtres et les pédagogues se sont secrètement approprié le monopole de l'élevage humain. Vallejo reprend la même idée lorsqu'il attaque l'Église catholique - puis l'Islam - avec une rare virulence, notamment dans son essai La puta de Babilonia. L'homme serait donc un «animal sous influence» soumis à deux formes d'éducation, l'une inhibante (la domestication par les textes philosophiques et littéraires) et l'autre désinhibante, menant à la bestialisation (la brutalité guerrière, l'ivresse déshumanisante des jeux du cirque chez les Romains et, plus près de nous, le hooliganisme et les Barras bravas des stades de foot ou le voyeurisme-exhibitionnisme de la télé-réalité, symbole de l'abêtissement quotidien par les médias). Dans La virgen de los sicarios, Vallejo montre clairement que l'humanité consiste à choisir entre ces deux influences, ces deux forces, symbolisées par le «grammairien» et les sicaires, les tueurs à gages. Le contexte décrit par Vallejo, dans ses romans et dans l'épitexte public est très comparable à celui de l'après-guerre en Europe: "Ese país vesánico se entregó en brazos de su concubina natural, la Violencia» (2002: 23). La Colombie, et plus particulièrement Medellín, apparaissent comme la métonymie allégorique de l'ensauvagement du monde.

Selon Sloterdijk, les nations modernes ne sont rien moins que « d'efficaces fictions nées d'une opinion lettrée rassemblée grâce à la lecture des mêmes auteurs». Ce formatage intellectuel dérive, à l'ère postmoderne, vers ce que Christian Salmon appelle le storytelling, discours triomphaliste et mensonger des politiciens: "Une tendance apparue dans les années 1980, sous la présidence de Ronald Reagan, lorsque les stories en vinrent à se substituer aux arguments raisonnés et aux statistiques dans les discours officiels» (Salmon 2006: 18-19). C'est cette pratique que Vallejo condamne sans cesse dans ses invectives contre des politiciens qui se comportent en stars des médias:

- ¡Y esta lora gárrula hablando hasta por los codos sin parar!

Se refería al presidente de su país que sólo gobierna cuatro años, pero bien hubiera podido referirse al nuestro, que gobierna seis [...]. Pues bien, con dos veces que viera el viejo a unos de esos granujas hablando por televisión y ya le quería cortar la lengua. Los detestaba. Y mientras más se hicieran ver, más, más los odiaba. Odiaba al primer ministro de Inglaterra, al presidente de Estados Unidos, al del gobierno español, al de Francia, a los déspotas de 
Cuba, Libia, Irak, Arabia, al demagogo de Venezuela, al capo vaticano, al energúmeno de Palestina, al juez Garzón (2002: 114-115).

La toute puissance des médias dans la culture de masse implique de nouvelles bases de coexistence au sein des sociétés postmodernes: "Ces bases sont, on peut sans peine le démontrer, résolument post-littéraires, post-épistolaires et, par conséquent, post-humanistes: la littérature, la correspondance et l'idéologie humaniste n'influencent plus aujourd'hui que marginalement les méga-sociétés modernes dans la production du lien politico-culturel»(Sloterdijk 2000). Dans les sociétés postmodernes, la littérature s'est marginalisée en une subculture souvent mise en échec par des médias inféodés au pouvoir économique et politique. Voilà le paradoxe qui s'impose à tout écrivain postmoderne: pourquoi et comment continuer à écrire si l'on admet l'échec de la culture écrite face à l'ensauvagement du monde et à la barbarie?

\section{Un virtuose de l'imprécation pour une mise en échec du storytelling}

Vallejo se situe dans cette perspective certes pessimiste, mais absolument pas nihiliste et encore moins réactionnaire, contrairement à ce qu'ont affirmé certains de ses détracteurs. Depuis la publication de son premier roman, Los días azules, en 1985, Vallejo constate l'échec de la démocratie colombienne. Condamnant les hypocrisies de l'Église catholique comme celles de l'intelligentsia de gauche, renvoyant dos à dos les partis de gauche comme de droite, l'écrivain colombien dénonce la perte des valeurs humanistes, l'incurie, le cynisme et la corruption généralisée. Mais il fustige aussi une population qui contemple machinalement le spectacle du monde, abêtie par la médiocrité des médias et aliénée par la société de consommation. Les imprécations de l'autofictionneur valléjien sapent les propos lénifiants et politiquement corrects des «moralistes» et autres «humanitaristes» et minent un discours «politicien» régi par la langue de bois et les affabulations du storytelling.

En réaction contre le conformisme intellectuel et social, Vallejo fonde une sorte d'exemplarité de la marginalité et de l'échec. Il ne s'agit pas d'ériger l'échec en modèle de conduite mais de rappeler que le mythe de la réussite, notamment matérielle, est une fiction - un «miroir embellisseur mensonger», dirait Hermann Broch - qui oublie et occulte la réalité des laissés-pour-compte, dissimulant aussi l'échec des politiciens à construire un monde meilleur. L'autofictionneur valléjien soulève le problème de la 
pauvreté et du populisme en Amérique latine mais il départicularise aussi la figure du pauvre pour l'actualiser dans celle du loser, c'est-à-dire du dominé.

Face à la défaite des idéologies et à la fictionnalisation du discours politique par les politiques eux-mêmes, l'affirmation de la première personne autofictionnelle, l'ambiguïté du pacte de lecture, le brouillage des genres (littéraires et sexuels), le stream of consciousness nostalgique et la prolifération imprécatoire parviennent à relever le défi de la fin de la littérature et à mettre le storytelling en échec.

\section{Bibliographie}

Deleuze, Gilles, 1983, L'image-mouvement, Cinéma 1, Paris, Les Éditions de Minuit. Deleuze, Gilles, 1985, L’image-temps, Cinéma 2, Paris, Les Éditions de Minuit.

Dulau, Pierre, 2008, Heidegger pas à pas, Paris, Ellipses.

Heidegger, Martin, 1957, Lettre sur l'humanisme, traduction française de Roger Munier, Paris, Éditions Montaigne.

Joset, Jacques, 2010, La muerte y la gramática. Los derroteros de Fernando Vallejo, Bogotá-Buenos Aires-Madrid-México, Taurus.

Larrañaga, Silvia, 2006, «Le brouillage de genres chez Fernando Vallejo. Le rôle de l'épitexte public», in Le texte et ses liens I / El texto y sus vínculos I, Cultures et littératures hispano-américaines, Les Ateliers du SAL, sous la direction de Milagros Ezquerro, textes réunis par Julien Roger, Paris, INDIGO \& côté-femmes éditions, $2^{\mathrm{e}}$ trimestre, p. 185-194.

Mengue, Philippe, 1994, Gilles Deleuze ou le système du multiple, "Cinéma 1 et 2 », Paris, Kimé.

Murillo, Javier H., «Un Huapiti para Fernando Vallejo», Revista Número, Bogotá, Colombia, non daté: http://www.revistanumero.com/16huapi.htm

Salmon, Christian, 2006, "Une machine à fabriquer des histoires", Le Monde Diplomatique, novembre, p. 18-19.

Salmon, Christian, 2007, Storytelling, La machine à fabriquer des histoires et à formater les esprits, Paris, éditions La Découverte.

Sloterdijk, Peter, 2000, Règles pour le parc humain. Une lettre en réponse à la Lettre sur l'Humanisme de Heidegger, traduit de l'allemand par Olivier Mannoni, Paris, Éditions Mille et Une nuits, «La petite collection». Publié sur le web par la revue Multitudes: http://multitudes.samizdat.net/Regles-pour-le-Parc-humain

Souquet, Lionel, 2009, "Ligne de fuite néo-picaresque dans l'autofiction hispanoaméricaine: Arenas, Copi, Lemebel, Vallejo», Hommage à Milagros Ezquerro, Théorie et fiction, México / París, RILMA 2 / ADEHL, p. 601-622. 
Souquet, Lionel, 2011, «Fernando Vallejo: Familles, je vous hais ! El desbarrancadero (2001), Mi hermano el alcalde (2004), El don de la vida (2010)», numéro spécial des Langues Néo-latines coordonné par Dorita Nouhaud, Le roman hispanoaméricain au XXIe siècle, 2: Des airs de famille, $\mathrm{n}^{\circ}$ 356, mars, p. 45-65.

Souquet, Lionel, 2011, «Je est l'Autre: archéologie de la construction identitaire et artistique de Reinaldo Arenas et de Fernando Vallejo», Amadis, n 9, «L'autre», Université de Bretagne Occidentale, Brest.

Souquet, Lionel, 2011, «Fernando Vallejo de Diógenes a Heidegger o el humanismo "cínico" del autoficcionador-filósofo", in Marián Semilla Durán, Des genres et du genre II - Fernando Vallejo: un noud de sens (contre toute imposture)» / "De los géneros y del género II - Fernando Vallejo, un nudo de sentido (contra toda impostura), Université Lumière Lyon 2.

Vallejo, Fernando, 1985a, Los días azules, Buenos Aires, Alfaguara.

Vallejo, Fernando, 1985b, Los caminos a Roma, Bogotá, Alfaguara.

Vallejo, Fernando, 1986, El fuego secreto, Bogotá, Alfaguara.

Vallejo, Fernando, 1991, El Mensajero, Una biografía de Porfirio Barba Jacob, Bogotá, Colombia, Alfaguara.

Vallejo, Fernando, 1993, Entre fantasmas, Buenos Aires, Alfaguara.

Vallejo, Fernando, 2001, El desbarrancadero, Madrid, Alfaguara.

Vallejo, Fernando, 2002, La rambla paralela, Buenos Aires, Alfaguara.

Vallejo, Fernando, 2004, Mi hermano el alcalde, Madrid, Alfaguara, Santillana Ediciones Generales.

Vallejo, Fernando, 2007, La puta de Babilonia, Barcelona, Seix Barral. 